Research Article

\title{
Vitamin K Status and Mobility Limitation and Disability in Older Adults: The Health, Aging, and Body Composition Study
}

\author{
M. Kyla Shea, PhD, ${ }^{1, *}$ Stephen B. Kritchevsky, PhD, ${ }^{2, \bullet}$ Richard F. Loeser, $\mathrm{MD}^{,}{ }^{3}$ and \\ Sarah L Booth, PhD'
}

'USDA Human Nutrition Research Center on Aging at Tufts University, Boston, Massachusetts. ${ }^{2}$ Sticht Center for Healthy Aging and Alzheimer's Prevention, Wake Forest School of Medicine, Winston-Salem, North Carolina. ${ }^{3}$ Thurston Arthritis Research Center, University of North Carolina School of Medicine, Chapel Hill, North Carolina.

*Address correspondence to: M. Kyla Shea, PhD, USDA Human Nutrition Research Center on Aging at Tufts University, Boston, MA. E-mail: kyla. shea@tufts.edu

Received: December 6, 2018; Editorial Decision Date: April 18, 2019

Decision Editor: David Melzer, MBBCh, PhD

\begin{abstract}
Background: Vitamin $\mathrm{K}$ has been implicated in chronic diseases associated with increased risk for mobility disability, such as osteoarthritis and cardiovascular disease. However, the association between vitamin $\mathrm{K}$ status and mobility disability is unknown. Therefore, we examined the association between vitamin $\mathrm{K}$ status and incident mobility disability in the Health, Aging, and Body Composition Study.

Methods: Plasma phylloquinone (vitamin K1) was categorized as $<0.5,0.5-<1.0$ and $\geq 1.0 \mathrm{nmol} / \mathrm{L}$ ( $n=1,323,48 \%$ male). Plasma ucMGP, which increases when vitamin K status is low, was measured in 716 participants and categorized into tertiles. Mobility limitation and disability, defined as two consecutive semiannual reports of having any or a lot of difficulty walking a one-fourth mile or climbing 10 steps without resting, were assessed over a median 6-10 years of follow-up. Multivariate Cox proportional hazard models were used to evaluate the association between vitamin $\mathrm{K}$ status and incident mobility limitation and disability.

Results: Participants with plasma phylloquinone less than $0.5 \mathrm{nmol} / \mathrm{L}$ were more likely to develop mobility limitation and disability compared to those with at least $1.0 \mathrm{nmol} / \mathrm{L}$ (adjusted HR (95\% CI) mobility limitation: 1.27 (1.05-1.53); disability: 1.34 (1.01-1.76)). After further adjustment for knee pain, the associations were partially attenuated (HR (95\% CI) mobility limitation: 1.20 (0.99-1.45); disability: 1.26 (0.96-1.67)). Plasma ucMGP was not associated with incident mobility limitation, but was nonlinearly associated with incident mobility disability (HR (95\% CI), compared to tertile 1 : tertile $2=1.64(1.19-2.27)$, tertile $3=1.17(0.83-1.66)$, fully adjusted).

Conclusion: Our results suggest vitamin $\mathrm{K}$ may be involved in the disablement process in older age. Future studies are needed to confirm our findings and clarify the underlying mechanism.
\end{abstract}

Keywords: Vitamin K, Matrix gla protein, Mobility, Disability, Aging.

Identifying novel risk factors for mobility disability is becoming increasingly important in order to deepen our understanding of its underlying pathophysiology and discover potential therapeutic targets. One potential novel risk factor is vitamin $\mathrm{K}$ status because vitamin $\mathrm{K}$ is implicated in chronic diseases that lead to disability (1). Vitamin $\mathrm{K}$ is an essential nutrient, so must be obtained from the diet or dietary supplements. The primary dietary form of vitamin $\mathrm{K}$ is phylloquinone, which is found in green leafy vegetables and vegetable oils. More than $60 \%$ of older men and $40 \%$ of older women do not meet the dietary recommendations for vitamin K (2). Vitamin $\mathrm{K}$ is therefore, considered a shortfall nutrient in older age and older adults with low vitamin $\mathrm{K}$ status may be more susceptible to vitamin K-related diseases and subsequent disability.

Vitamin K's main function is as an enzymatic co-factor in the post-translational carboxylation of vitamin K-dependent proteins, several of which are mechanistically linked to musculoskeletal and other chronic diseases (3). We and others found low vitamin $\mathrm{K}$ status was associated with higher risk of osteoarthritis $(\mathrm{OA})$ and 
cardiovascular disease (CVD) (1,4-6). We also previously found older adults (from the Health, Aging, and Body Composition Study [Health $\mathrm{ABC}]$ ) with lower plasma phylloquinone had slower gait speed and worse physical performance over 4-5 years of follow-up (7). Collectively these findings suggest vitamin K status could be associated with mobility disability, but this has never been evaluated formally. The aim of the present study, therefore, was to evaluate the association of plasma phylloquinone and uncarboxylated matrix gla protein, two biomarkers of vitamin $\mathrm{K}$ status, with incident mobility limitation and disability in Health ABC.

\section{Methods}

Participants were drawn from Health ABC, a prospective cohort designed to examine the associations of body composition, weightrelated health conditions, and mobility in 3,075 older black and white adults (8). When enrolled, all participants were 70-79 years old, free of self-reported disability in activities of daily living, difficulty walking one-fourth mile or up 10 steps without resting.

\section{Vitamin K Status}

Blood samples were taken at the year 2 clinic visit (1998/1999) after an overnight fast and stored at $-70^{\circ} \mathrm{C}$ until time of analysis. Plasma phylloquinone (vitamin K1) was measured using HPLC in 1,127 participants in the Health $\mathrm{ABC}$ Knee Osteoarthritis substudy and in 731 randomly selected participants who were not included in the Knee Osteoarthritis substudy, as described (9). Plasma dephosphouncarboxylated MGP (ucMGP) was measured in participants in the knee OA substudy only ( $n=1,127)$, using a sandwich enzyme-linked immunosorbent assay, which uses two monoclonal antibodies directed against the dephosphorylated amino acid sequence 3-15 and the uncarboxylated amino acid sequence 35-49 in human MGP. The reported intra- and inter-assay variability was $5.6 \%$ and $9.9 \%$, respectively (10). Lower ucMGP reflects higher vitamin K status (11).

\section{Mobility Limitation and Disability}

Mobility was assessed every 6 months during annual clinic visits and interim telephone interviews $(8,12)$. For the present analysis, we evaluated:

(1) Mobility limitation: defined as two consecutive semiannual reports of having any difficulty either walking a one-fourth mile or climbing 10 steps without resting $(8,12)$.

(2) Mobility disability: defined as two consecutive semiannual reports of having a lot of difficulty or inability walking a onefourth mile or climbing 10 steps without resting $(8,12)$.

The year 2 clinic visit was considered baseline, corresponding to when vitamin $\mathrm{K}$ status was measured. Participants who had developed mobility limitation by the year 2 clinic visit were not included $(n=425)$. Warfarin is a vitamin $\mathrm{K}$ antagonist, so warfarin users were also excluded $(n=110)$. There were 1,323 participants available for inclusion in the analysis of plasma phylloquinone, 716 of whom had measures of plasma ucMGP, so were available for that analysis as well. The median (quartile range) follow-up was 6.4 (8.6) years and 10.3 (5.8) years for incident mobility limitation and mobility disability, respectively.

Compared to those included, participants excluded because they developed mobility limitation by their year 2 clinic visit and/or because they used warfarin were more likely to be female, black, have knee pain, prevalent CVD, and hypertension (all $p<.001$ ), and less likely to have graduated from college $(p<.001)$.

\section{Covariates}

The following covariates were measured as described in the Supplementary Methods: age, sex, race, body mass index, education, triglycerides, creatinine, interleukin-6, cognitive status, smoking status, healthy eating index, medication use.

\section{Statistical Approach}

A detailed description of the statistical approach is provided in the Supplementary Material, and summarized here.

Plasma phylloquinone was categorized as $\geq 1.0 \mathrm{nmol} / \mathrm{L}, 0.5-$ $<1.0 \mathrm{nmol} / \mathrm{L}$ and $<0.5 \mathrm{nmol} / \mathrm{L}$. These categories are based on the following: When the recommended Adequate Intakes for vitamin $\mathrm{K}$ are met, circulating phylloquinone approximates $1.0 \mathrm{nmol} / \mathrm{L}(13,14)$. A concentration less than $0.5 \mathrm{nmol} / \mathrm{L}$ would correspond to dietary vitamin $\mathrm{K}$ intakes less than half of the recommended Adequate Intakes. As the ucMGP concentration corresponding to vitamin $\mathrm{K}$ dietary recommendations is not known, it was categorized according to tertiles, with the lowest tertile reflecting higher vitamin $\mathrm{K}$ status.

Cox proportional hazard regression was used to evaluate the association of vitamin $\mathrm{K}$ status with incident mobility limitation and disability. Adjusted models controlled for age, race, body mass index, highest level of education, study site, triglycerides (because phylloquinone is transported on triglyceride-rich lipoproteins), IL-6, prevalent cardiovascular, hypertension, kidney function (estimated glomerular filtration rate), and cognitive function (Mini Mental Status Exam score). The fully adjusted models additionally controlled for healthy eating index and smoking status. We added knee pain, a common symptom of knee OA, as a separate covariate to the fully adjusted model. The cross-sectional association between vitamin $\mathrm{K}$ status and mobility limitation and disability at the year 2 clinic visit was evaluated using logistic regression. All analyses were carried out using SAS, v 9.4.

\section{Results}

The study population consisted of 635 men and 688 women, with a mean $\pm S D$ age of $74.6 \pm 2.8$ years. Forty per cent were black. The descriptive characteristics according to plasma phylloquinone and ucMGP categories in are shown in Tables 1 and 2. Plasma phylloquinone was positively associated with triglycerides and the Healthy Eating Index, and inversely associated with IL-6 and knee pain (Table 1). Plasma ucMGP was positively associated with triglycerides and IL-6 and inversely associated with estimated glomerular filtration rate. Black participants were also more likely to have lower ucMGP (Table 2).

A total of 902 participants reported incident mobility limitation and 409 reported mobility disability during follow-up. Those with less than $0.5 \mathrm{nmol} / \mathrm{L}$ plasma phylloquinone were significantly more likely to develop mobility limitation and disability, compared to those with at least $1.0 \mathrm{nmol} / \mathrm{L}$. However, after adjustment for knee pain, the hazard for mobility disability did not significantly differ between those with plasma phylloquinone less than $0.5 \mathrm{nmol} / \mathrm{L}$ and those with at least $1.0 \mathrm{nmol} / \mathrm{L}$ (Table 3).

Plasma ucMGP was not associated with incident mobility limitation. However, plasma ucMGP was associated with mobility disability such that those in the middle ucMGP tertile were more likely to develop mobility disability compared to those in the lowest tertile, 
Table 1. Baseline Characteristics of Health ABC Participants According to Plasma Phylloquinone Categories*

\begin{tabular}{|c|c|c|c|c|}
\hline & $<0.5 \mathrm{nmol} / \mathrm{L}(n=250)$ & $0.5-<1.0 \mathrm{nmol} / \mathrm{L}(n=485)$ & $\geq 1.0 \mathrm{nmol} / \mathrm{L}(n=588)$ & $p^{\dagger}$ \\
\hline Age $(y)$ & $74.8 \pm 2.9$ & $74.6 \pm 2.9$ & $74.4 \pm 2.9$ & .249 \\
\hline Male, $n(\%)$ & $120(48)$ & $250(52)$ & $265(45)$ & .107 \\
\hline Black, $n(\%)$ & $118(47)$ & $185(38)$ & $228(39)$ & .070 \\
\hline Pittsburgh, $n(\%)$ & $147(59)$ & $253(52)$ & $272(46)$ & .003 \\
\hline \multirow{3}{*}{$\begin{array}{l}\text { Education, } n(\%) \leq \text { high school } \\
>\text { high school }<\text { college } \\
\geq \text { college }\end{array}$} & $76(31)$ & $96(20)$ & $131(22)$ & \multirow[t]{3}{*}{.002} \\
\hline & $88(35)$ & $152(31)$ & $187(32)$ & \\
\hline & $85(34)$ & $236(49)$ & $270(46)$ & \\
\hline $\mathrm{BMI}\left(\mathrm{kg} / \mathrm{m}^{2}\right)$ & $26.4 \pm 4.4$ & $26.7 \pm 4.5$ & $27.5 \pm 4.5$ & $<.001$ \\
\hline Triglycerides $(\mathrm{mg} / \mathrm{dL})^{\ddagger}$ & $109 \pm 66$ & $116 \pm 59$ & $132 \pm 92$ & $<.001$ \\
\hline $\mathrm{IL}-6, \mathrm{pg} / \mathrm{mL}^{\ddagger}$ & $2.6 \pm 2.3$ & $2.2 \pm 2.1$ & $2.1 \pm 2.1$ & $<.001$ \\
\hline \multicolumn{5}{|l|}{ Prevalent health conditions } \\
\hline Cardiovascular disease, $n(\%)$ & $48(19)$ & $85(18)$ & $115(20)$ & .683 \\
\hline Hypertension, $n(\%)$ & $116(46)$ & $236(49)$ & $329(56)$ & .012 \\
\hline Knee pain, $n(\%)$ & $129(52)$ & $193(40)$ & $213(36)$ & .002 \\
\hline $3 \mathrm{MS}$ score $^{\ddagger}$ & $91 \pm 11$ & $93 \pm 8$ & $93 \pm 9$ & .001 \\
\hline Smoker, $n(\%)$ & $16(13)$ & $24(10)$ & $21(8)$ & .031 \\
\hline Healthy eating index & $64.4 \pm 12.6$ & $69.8 \pm 12.2$ & $70.5 \pm 11.9$ & .004 \\
\hline eGFR & $70.4 \pm 14.9$ & $68.3 \pm 14.0$ & $68.7 \pm 15.2$ & .171 \\
\hline $\begin{array}{l}\text { ucMGP, } \mathrm{pmol} / \mathrm{L}^{\ddagger} \\
(n=716)\end{array}$ & $482 \pm 483$ & $438 \pm 432$ & $381 \pm 426$ & .004 \\
\hline
\end{tabular}

Notes: $\mathrm{BMI}=$ body mass index; eGFR = estimated glomerular filtration rate; $3 \mathrm{MS}=$ Mini Mental Status Exam.

*Data presented as mean $\pm S D$, unless indicated otherwise.

${ }^{\dagger}$ Based on analysis of variance (continuous measures) or chi-square test (categorical measures), unless indicated otherwise.

敋ata presented as median $\pm \mathrm{IQR}, p$-value based on Kruskal-Wallis test.

Table 2. Baseline Characteristics of Health ABC Participants According to Plasma ucMGP Tertiles*

\begin{tabular}{|c|c|c|c|c|}
\hline & $\begin{array}{l}\text { Tertile } 1(<297 \\
\mathrm{pmol} / \mathrm{L} ; n=245)\end{array}$ & $\begin{array}{l}\text { Tertile } 2(298-582 \\
\mathrm{pmol} / \mathrm{L} ; n=242)\end{array}$ & $\begin{array}{l}\text { Tertile } 3(\geq 583 \\
\mathrm{pmol} / \mathrm{L} ; n=229)\end{array}$ & $p^{\dagger}$ \\
\hline Age $(y)$ & $74.3 \pm 2.7$ & $74.5 \pm 2.9$ & $74.8 \pm 2.9$ & .143 \\
\hline Male, $n(\%)$ & $106(43)$ & $107(44)$ & $106(46)$ & .797 \\
\hline Black, $n(\%)$ & $134(55)$ & $103(43)$ & $65(28)$ & $<.001$ \\
\hline Pittsburgh, $n(\%)$ & $105(43)$ & $134(55)$ & $142(62)$ & $<.001$ \\
\hline Education, $n(\%) \leq$ high school & $63(26)$ & $60(25)$ & $38(17)$ & .036 \\
\hline$>$ high school $<$ college & $71(29)$ & $73(30)$ & $91(40)$ & \\
\hline$\geq$ college & $111(45)$ & $109(40)$ & $100(44)$ & \\
\hline $\operatorname{BMI}\left(\mathrm{kg} / \mathrm{m}^{2}\right)$ & $27.0 \pm 4.6$ & $27.1 \pm 4.4$ & $27.7 \pm 4.6$ & .159 \\
\hline Triglycerides $(\mathrm{mg} / \mathrm{dL})^{\ddagger}$ & $112 \pm 67$ & $122 \pm 62$ & $135 \pm 78$ & $<.001$ \\
\hline $\mathrm{IL}-6, \mathrm{pg} / \mathrm{mL}^{\ddagger}$ & $2.1 \pm 2.1$ & $2.3 \pm 2.2$ & $2.5 \pm 2.4$ & .034 \\
\hline \multicolumn{5}{|l|}{ Prevalent health conditions } \\
\hline Cardiovascular disease, $n(\%)$ & $41(17)$ & $42(17)$ & $58(25)$ & .034 \\
\hline Hypertension, $n(\%)$ & $130(53)$ & $124(52)$ & $120(52)$ & .954 \\
\hline Knee pain, $n(\%)$ & $169(69)$ & $166(69)$ & $157(69)$ & .994 \\
\hline $3 \mathrm{MS}$ score $^{\ddagger}$ & $92 \pm 10$ & $92 \pm 8$ & $94 \pm 7$ & .055 \\
\hline Smoker, $n(\%)$ & $30(12)$ & $18(8)$ & $13(6)$ & .030 \\
\hline Healthy eating index & $69.1 \pm 12.0$ & $70.2 \pm 12.3$ & $68.8 \pm 11.4$ & .588 \\
\hline eGFR & $72.5 \pm 14.4$ & $69.3 \pm 14.1$ & $65.3 \pm 13.3$ & $<.001$ \\
\hline Plasma phylloquinone, $\mathrm{nmol} / \mathrm{L}^{\ddagger}$ & $0.8 \pm 1.0$ & $0.8 \pm 0.7$ & $0.7 \pm 0.6$ & .002 \\
\hline
\end{tabular}

Notes: $\mathrm{BMI}=$ body mass index; eGFR = estimated glomerular filtration rate; $3 \mathrm{MS}=$ Mini Mental Status Exam.

*Data presented as mean $\pm S D$, unless indicated otherwise.

${ }^{\dagger}$ Based on analysis of variance (continuous measures) or chi-square test (categorical measures), unless indicated otherwise.

${ }^{\ddagger}$ Data presented as median $\pm \mathrm{IQR}, p$-value based on Kruskal-Wallis test.

but there was no difference in incident mobility disability between those in the highest and lowest tertiles (Table 3).

When mobility disability and death were analyzed as a composite outcome, the results were similar to the analyses of mobility disability alone (Supplementary Table 1). When analyses of plasma phylloquinone and mobility were limited to the subset of participants who had ucMGP measures $(n=716)$, the association between plasma phylloquinone and mobility limitation was similar, but the association with mobility disability was attenuated (Supplementary Table 2). Substitution of knee pain with Kellgren-Lawrence grade, 
Table 3. Hazard Ratio (95\% Confidence Interval) for Mobility Limitation and Disability According to Vitamin K Status in the Health ABC Study

\begin{tabular}{|c|c|c|c|c|c|}
\hline & Events $/ n$ & $\begin{array}{l}\text { Plasma Phylloquinone } \\
<0.5 \mathrm{nmol} / \mathrm{L}\end{array}$ & $\begin{array}{l}\text { Plasma Phylloquinone } \\
0.5-<1.0 \mathrm{nmol} / \mathrm{L}\end{array}$ & $\begin{array}{l}\text { Plasma Phylloquinone } \geq \\
1.0 \mathrm{nmol} / \mathrm{L}\end{array}$ & $p$-Trend* \\
\hline \multicolumn{6}{|c|}{ Incident mobility limitation } \\
\hline Unadjusted & $902 / 1323$ & $1.23(1.03-1.46)$ & $1.05(0.90-1.21)$ & 1.00 & .033 \\
\hline Model $2^{\dagger}$ & $847 / 1247$ & $1.27(1.05-1.53)$ & $1.15(0.98-1.34)$ & 1.00 & .008 \\
\hline Model $3^{\ddagger}$ & $819 / 1216$ & $1.27(1.05-1.53)$ & $1.12(0.95-1.31)$ & 1.00 & .013 \\
\hline $\begin{array}{l}\text { Additionally adjusted } \\
\text { for knee pain }\end{array}$ & $818 / 1215$ & $1.20(0.99-1.45)$ & $1.11(0.94-1.30)$ & 1.00 & .051 \\
\hline \multicolumn{6}{|l|}{ Incident mobility disability } \\
\hline Unadjusted & $409 / 1322$ & $1.14(0.88-1.47)$ & $1.03(0.83-1.28)$ & 1.00 & .357 \\
\hline Model $2^{\dagger}$ & $388 / 1247$ & $1.31(1.00-1.73)$ & $1.10(0.87-1.38)$ & 1.00 & .057 \\
\hline Model $3^{\ddagger}$ & $376 / 1220$ & $1.34(1.01-1.76)$ & $1.08(0.85-1.37)$ & 1.00 & .053 \\
\hline \multirow[t]{2}{*}{$\begin{array}{l}\text { Additionally adjusted } \\
\text { for knee pain }\end{array}$} & $376 / 1220$ & $1.26(0.96-1.67)$ & $1.03(0.82-1.31)$ & 1.00 & .127 \\
\hline & Events $/ n$ & Plasma ucMGP $<297 \mathrm{pmol} / \mathrm{L}$ & Plasma ucMGP 297-582 pmol/L & Plasma ucMGP $\geq 583 \mathrm{pmol} / \mathrm{L}$ & $p$-Trend* \\
\hline \multicolumn{6}{|c|}{ Incident mobility limitation } \\
\hline Unadjusted & $523 / 716$ & 1.00 & $0.96(0.78-1.19)$ & $1.01(0.82-1.24)$ & .960 \\
\hline Model $2^{+}$ & $493 / 675$ & 1.00 & $0.94(0.75-1.17)$ & $0.96(0.77-1.21)$ & .744 \\
\hline Model $3^{\ddagger}$ & $484 / 663$ & 1.00 & $0.96(0.77-1.20)$ & $0.99(0.78-1.25)$ & .948 \\
\hline $\begin{array}{l}\text { Additionally adjusted } \\
\text { for knee pain }\end{array}$ & $484 / 663$ & 1.00 & $0.99(0.79-1.24)$ & $1.03(0.81-1.31)$ & .781 \\
\hline \multicolumn{6}{|l|}{ Incident mobility disability } \\
\hline Unadjusted & $254 / 715$ & 1.00 & $1.43(1.06-1.94)$ & $1.10(0.86-1.39)$ & $.031^{s}$ \\
\hline Model $2^{\dagger}$ & $243 / 674$ & 1.00 & $1.51(1.10-2.07)$ & $1.11(0.78-1.56)$ & $.006^{\S}$ \\
\hline Model $3^{\ddagger}$ & $237 / 658$ & 1.00 & $1.58(1.14-2.18)$ & $1.15(0.81-1.63)$ & $.003^{s}$ \\
\hline $\begin{array}{l}\text { Additionally adjusted } \\
\text { for knee pain }\end{array}$ & $237 / 658$ & 1.00 & $1.64(1.19-2.27)$ & $1.17(0.83-1.66)$ & $.001^{\mathrm{s}}$ \\
\hline
\end{tabular}

Notes: *Trend was tested using an ordinal variable for plasma phylloquinone category and ucMGP tertile.

${ }^{\dagger}$ Adjusted for age, race, site, body mass index, education, triglycerides, IL-6, eGFR, prevalent cardiovascular disease, hypertension, cognitive function (3MS score).

${ }^{\ddagger}$ Adjusted for covariates in model 2 plus healthy eating index, smoking status.

${ }^{\circledR}$ Model also included quadratic term (ucMGP tertile $\times$ ucMGP tertile), all quadratic term $p \leq .045$.

an indicator of structural knee OA severity, as a covariate in the model did not change the results (data not shown).

When analyzed cross-sectionally, participants with less than $0.5 \mathrm{nmol} / \mathrm{L}$ plasma phylloquinone were 1.49 times more likely to have mobility limitation (OR (95\% CI) $=1.49$ (1.04-2.13), fully adjusted) and nearly twice as likely to have mobility disability (OR $(95 \% \mathrm{CI})=1.95(1.08-3.54)$, fully adjusted $)$ compared to those with at least $1.0 \mathrm{nmol} / \mathrm{L}$. The odds for mobility limitation and disability did not differ significantly between those with $0.5-1.0 \mathrm{nmol} / \mathrm{L}$ and those with at least $1.0 \mathrm{nmol} / \mathrm{L}$ (OR (95\% CI) mobility limitation $=1.19(0.87-1.63)$, mobility disability $=1.65(0.97-2.81)$, fully adjusted). The odds of having mobility limitation or disability did not differ significantly across ucMGP tertiles (OR (95\% CI), compared to tertile 1 , mobility limitation: tertile $2=1.16(0.77-1.74)$, tertile $3=1.42(0.93-2.17)$; mobility disability: tertile $2=0.88$ $(0.44-1.74)$, tertile $3=1.62(0.84-3.13)$, all fully adjusted).

\section{Discussion}

Our hypothesis that vitamin $\mathrm{K}$ status is associated with mobility limitation and disability was motivated by prior observations that low plasma phylloquinone has been associated with a higher risk of chronic diseases that can result in mobility disability $(4,5,9,15)$. In Health $\mathrm{ABC}$, we previously found low vitamin $\mathrm{K}$ status was associated with worse lower-extremity performance over 4-5 years of follow-up (7). In our current analysis, we found older adults with plasma phylloquinone less than $0.5 \mathrm{nmol} / \mathrm{L}$ were more likely to develop mobility limitation and disability compared to those with at least $1.0 \mathrm{nmol} / \mathrm{L}$. Adjustment for knee pain, the primary symptom of knee OA, attenuated the association somewhat, but not completely. This suggests knee OA symptoms did not largely account for the association between plasma phylloquinone and mobility limitation or disability. Low vitamin $\mathrm{K}$ status has also been associated with CVD and inflammation $(1,9,16,17)$, which have also been linked to mobility disability $(12,18)$. However, our models controlled for CVD and IL-6, so the association between plasma phylloquinone and mobility limitation and disability appears to be independent of these potential mediators as well.

Plasma ucMGP was associated with mobility disability but not with mobility limitation. Furthermore, the association with mobility disability was nonlinear, such that those in the middle tertile were significantly more likely to develop mobility disability compared to those in the lowest tertile, but the hazard for mobility disability did not differ significantly between the highest and lowest tertiles. We do not have a clear explanation for why the hazard would be highest in the middle group. In this same cohort, plasma ucMGP was similarly related to worsening of articular cartilage damage and subchondral bone loss, two structural features of knee OA (4). However, adjustment for these variables did not change our findings (data not shown). Others reported an inverse association between plasma ucMGP and physical function in women, but not men (19). However, in Health $\mathrm{ABC}$, ucMGP was not consistently associated with lower-extremity function, and this association was not modified by sex (7). The current collective evidence regarding ucMGP and lower-extremity function is inconsistent. Well-designed mechanistic 
experiments focused on understanding the role of MGP in physiological processes involved in function and mobility are needed.

It is also plausible vitamin K's role in mobility is through mechanisms unrelated to its role as an enzyme co-factor. In recent in vitro experiments, bovine skeletal muscle cells treated with menaquinone-4, which is a metabolite of phylloquinone, exhibited increased cell migration and myogenic transcription factor expression during cell proliferation, but not during cell differentiation (20). This suggests there could be a direct effect of vitamin $\mathrm{K}$ on skeletal muscle that does not involve the carboxylation of vitamin K-dependent proteins. This may also explain why the association of plasma phylloquinone with mobility limitation/disability was not consistent with the association of plasma ucMGP with mobility limitation/disability.

To the best of our knowledge, this study is the first to evaluate the association between biomarkers of vitamin $\mathrm{K}$ status and mobility limitation and disability in older adults. In Health ABC, mobility was evaluated every 6 months and Health $\mathrm{ABC}$ participants were well characterized for potential confounders, which are notable strengths. Defining mobility limitation and disability using two consecutive reports reduced the likelihood of misclassifying temporary injuries as persistent disability. However, the observational design limits the ability to infer causation. Plasma phylloquinone and ucMGP were only available at one time point, and repeated measures would provide a more reliable estimate of vitamin $\mathrm{K}$ status over the long term. There is not a clinical definition for vitamin $\mathrm{K}$ deficiency based on biomarkers because the relevance of different thresholds to clinical endpoints has not been studied extensively (21). Circulating ucMGP is reduced by vitamin K supplementation $(11,22)$, and lower ucMGP reflects better vitamin $\mathrm{K}$ status. However, circulating ucMGP also depends on the synthesis of MGP, which is not dependent on vitamin K. We previously found total MGP (which is measured regardless of its carboxylation status) was a significant predictor of ucMGP (11). However, we were unable to adjust our models or express ucMGP as a ratio of total MGP, which is the practice with other vitamin $\mathrm{K}$ dependent proteins (23), because total MGP was not measured in Health ABC. Circulating phylloquinone positively correlates with triglycerides because phylloquinone is transported on triglyceride-rich lipoproteins. In Health ABC, plasma ucMGP also positively correlated with triglycerides, even though plasma phylloquinone and ucMGP were inversely associated. MGP synthesis can be upregulated in the setting of certain chronic diseases such as CVD. The positive association between ucMGP and triglycerides might reflect a mutual positive correlation with cardiovascular or other chronic diseases. In our previous analysis of lower-extremity function, Health $\mathrm{ABC}$ participants with at least $1.0 \mathrm{nmol} / \mathrm{L}$ plasma phylloquinone had better function compared to those with less than $1.0 \mathrm{nmol} / \mathrm{L}$, and we did not detect effect modification by sex (7). In the current study, those with less than $0.5 \mathrm{nmol} / \mathrm{L}$ were most at risk for mobility limitation and disability. This could indicate a lower threshold applies to mobility limitation or disability compared to individual tasks of lower-extremity function. Because the participants excluded for developing mobility limitation prior to the year 2 clinic visit or for warfarin use were less healthy than the included participants, our findings may not be generalizable to less healthy groups. The association between plasma phylloquinone and mobility disability was attenuated when we limited the analysis to the subset of participants in whom ucMGP was also measured, so the generalizability of those findings is also questionable. However, the association with mobility limitation was generally consistent. This could indicate vitamin $\mathrm{K}$ is involved earlier in the disablement process.
The population of adults at least 65 years old in the United States is projected to nearly double by 2050 (24), and the burden of mobility limitation will increase concomitantly. Identifying novel risk factors is important to help reduce the individual and public health burden of mobility disability. Our findings suggest vitamin $\mathrm{K}$ may be involved in the disablement process. If confirmed in future studies, vitamin $\mathrm{K}$ supplementation may represent a novel and straightforward strategy to help maintain mobility and independence in older age.

\section{Supplementary Material}

Supplementary data are available at The Journals of Gerontology, Series A: Biological Sciences and Medical Sciences online.

\section{Funding}

Supported by the National Institute of Arthritis and Musculoskeletal and Skin Diseases (K01AR063167 and R21AR062284), the Arthritis Foundation (new investigator grant), the National Institute on Aging Intramural Research Program (N01AG62101, N01AG62103, N01AG62106, and R01AG028050), the National Institute of Nursing Research (R01NR012459), the Wake Forest Older Americans Independence Center (P30AG021332), and the United States Department of Agriculture (USDA), Agricultural Research Service (ARS) Cooperative Agreement (5819507707). Any opinions, findings, conclusions, or recommendations expressed in this publication are those of the authors and do not necessarily reflect the view of the USDA.

\section{Conflict of interest statement}

None reported.

\section{References}

1. Harshman SG, Shea MK. The role of vitamin $\mathrm{K}$ in chronic aging diseases: inflammation, cardiovascular disease, and osteoarthritis. Curr Nutr Rep. 2016;5:90-98. doi:10.1007/s13668-016-0162-x

2. Harshman SG, Finnan EG, Barger KJ, et al. Vegetables and mixed dishes are top contributors to phylloquinone intake in US adults: data from the 2011-2012 NHANES. J Nutr. 2017;147(7):1308-1313. doi:10.3945/ jn.117.248179

3. McCann JC, Ames BN. Vitamin K, an example of triage theory: is micronutrient inadequacy linked to diseases of aging? Am J Clin Nutr. 2009;90:889-907. doi:10.3945/ajcn.2009.27930

4. Shea MK, Kritchevsky SB, Hsu FC, et al.; Health ABC Study. The association between vitamin $\mathrm{K}$ status and knee osteoarthritis features in older adults: the Health, Aging and Body Composition Study. Osteoarthritis Cartilage. 2015;23:370-378. doi:10.1016/j.joca.2014.12.008

5. Misra D, Booth SL, Tolstykh I, et al. Vitamin K deficiency is associated with incident knee osteoarthritis. Am J Med. 2013;126:243-248. doi:10.1016/j.amjmed.2012.10.011

6. Zhang S, Guo L, Bu C. Vitamin K status and cardiovascular events or mortality: a meta-analysis. Eur J Prev Cardiol. 2019;26:549-553. doi:10.1177/2047487318808066

7. Shea MK, Loeser RF, Hsu FC, et al.; Health ABC Study. Vitamin K status and lower extremity function in older adults: the Health Aging and Body Composition Study. J Gerontol A Biol Sci Med Sci. 2016;71:1348-1355. doi:10.1093/gerona/glv209

8. Houston DK, Neiberg RH, Tooze JA, et al.; Health ABC Study. Low 25-hydroxyvitamin D predicts the onset of mobility limitation and disability in community-dwelling older adults: the Health ABC Study. J Gerontol A Biol Sci Med Sci. 2013;68:181-187. doi:10.1093/gerona/ gls136

9. Shea MK, Booth SL, Weiner DE, et al.; Health ABC Study. Circulating vitamin $\mathrm{K}$ is inversely associated with incident cardiovascular disease 
risk among those treated for hypertension in the Health, Aging, and Body Composition Study (Health ABC). J Nutr. 2017;147:888-895. doi:10.3945/jn.117.249375

10. Cranenburg EC, Koos R, Schurgers LJ, et al. Characterisation and potential diagnostic value of circulating matrix Gla protein (MGP) species. Thromb Haemost. 2010;104:811-822. doi:10.1160/TH09-11-0786

11. Shea MK, O'Donnell CJ, Vermeer C, et al. Circulating uncarboxylated matrix gla protein is associated with vitamin $\mathrm{K}$ nutritional status, but not coronary artery calcium, in older adults. J Nutr. 2011;141:1529-1534. doi:10.3945/jn.111.139634

12. Cesari M, Kritchevsky SB, Nicklas B, et al.; Health ABC study. Oxidative damage, platelet activation, and inflammation to predict mobility disability and mortality in older persons: results from the health aging and body composition study. J Gerontol A Biol Sci Med Sci. 2012;67:671-676. doi:10.1093/gerona/glr246

13. Booth SL, Martini L, Peterson JW, Saltzman E, Dallal GE, Wood RJ. Dietary phylloquinone depletion and repletion in older women. $J$ Nutr. 2003;133:2565-2569. doi:10.1093/jn/133.8.2565

14. Food and Nutrition Board IoM. Dietary Reference Intakes for Vitamin A, Vitamin K, Arsenic, Boron, Chromium, Copper, Iodine, Iron, Manganese, Molybdenum, Nickel, Silicon, Vanadium, and Zinc. Washington DC: National Academies Press; 2001.

15. Wen L, Chen J, Duan L, Li S. Vitamin K-dependent proteins involved in bone and cardiovascular health (review). Mol Med Rep. 2018;18:3-15. doi:10.3892/mmr.2018.8940

16. Shea MK, Booth SL, Massaro JM, et al. Vitamin K and vitamin D status: associations with inflammatory markers in the Framingham Offspring Study. Am J Epidemiol. 2008;167:313-320. doi:10.1093/ aje/kwm306
17. Shea MK, Cushman M, Booth SL, Burke GL, Chen H, Kritchevsky SB. Associations between vitamin $\mathrm{K}$ status and haemostatic and inflammatory biomarkers in community-dwelling adults. The Multi-Ethnic Study of Atherosclerosis. Thromb Haemost. 2014;112:438-444. doi:10.1160/ TH13-12-1003

18. Heiland EG, Qiu C, Wang R, et al. Cardiovascular risk burden and future risk of walking speed limitation in older adults. J Am Geriatr Soc. 2017;65:2418-2424. doi:10.1111/jgs.15158

19. van Ballegooijen AJ, van Putten SR, Visser M, Beulens JW, Hoogendijk EO. Vitamin K status and physical decline in older adults-the Longitudinal Aging Study Amsterdam. Maturitas. 2018;113:73-79. doi:10.1016/j. maturitas.2018.04.013

20. Rønning SB, Pedersen ME, Berg RS, Kirkhus B, Rødbotten R. Vitamin K2 improves proliferation and migration of bovine skeletal muscle cells in vitro. PLoS One. 2018;13:e0195432. doi:10.1371/journal.pone.0195432

21. Shea MK, Booth SL. Concepts and controversies in evaluating vitamin K status in population-based studies. Nutrients. 2016;8(1). doi:10.3390/ nu8010008

22. Theuwissen E, Cranenburg EC, Knapen MH, et al. Low-dose menaquinone-7 supplementation improved extra-hepatic vitamin $\mathrm{K}$ status, but had no effect on thrombin generation in healthy subjects. Br J Nutr. 2012;108:1652-1657. doi:10.1017/S0007114511007185

23. Gundberg CM, Nieman SD, Abrams S, Rosen H. Vitamin K status and bone health: an analysis of methods for determination of undercarboxylated osteocalcin. J Clin Endocrinol Metab. 1998;83:3258-3266. doi:10.1210/ jcem.83.9.5126

24. Ortman JM, Velkhoff VA. An Aging Nation: The Older Population in the United States. Washington, DC: U.S. Department of Commerce Economics and Statistics Administration; 2014. 